\title{
Burden of Pneumonia Among Hospitalized Patients with Influenza: Real-World Evidence from a US Managed Care Population
}

\author{
Susan C. Bolge · Cynthia Gutierrez · Furaha Kariburyo • \\ Ding $\mathrm{He}$
}

Received: June 18, 2021 / Accepted: July 29, 2021 / Published online: August 16, 2021

(C) The Author(s) 2021

\section{ABSTRACT}

Introduction: Pneumonia is among the most prevalent complications of influenza. The purpose of this study is to quantify the burden of pneumonia among hospitalized patients with influenza.

Methods: Real-world retrospective data from 01JAN2014-30JUN2019 (study period) were obtained from Optum's de-identified Clinformatics ${ }^{\circledR}$ Data Mart Database (2007-2020) for patients who had $\geq 1$ diagnosis for influenza during the identification period and $\geq 1$ allcause inpatient visit within 1 day of diagnosis. Cases had $\geq 1$ diagnosis claim for an influenzarelated pneumonia within the 30 days after the initial influenza diagnosis date. Controls had no evidence of influenza-related pneumonia in the 30 days following the initial influenza diagnosis. Final 1:1 matching was determined using

Ding He and Furaha Kariburyo were employed at the time of the study.

Supplementary Information The online version contains supplementary material available at https:// doi.org/10.1007/s41030-021-00169-2.

S. C. Bolge

Janssen Global Services, LLC, Raritan, NJ, USA

C. Gutierrez $(\bowtie) \cdot$ F. Kariburyo · D. He

SIMR, LLC, 4110 Varsity Dr, Ann Arbor, MI 48108 , USA

e-mail: cgutierrez@statinmed.com propensity score matching (PSM). Statistical significance between the cohorts was tested.

Results: After PSM, there were 4878 hospitalized patients with influenza in each of the case and control groups. During the index hospitalization, cases vs. controls had longer length of stay [Mean (standard deviation): 6.5 (8.3) vs. 1.9 (3.7)], greater intensive care unit (ICU) use (38.4 vs. $16.8 \%$ ), and greater mechanical ventilation use (invasive: 11.4 vs. $2.3 \%$; non-invasive: 6.8 vs. $2.6 \%$ ) (all $p<0.001$ ). Cases also had higher readmission rates than controls (12.3 vs. $3.5 \%$ within 30 days; 20.0 vs. $6.1 \%$ within 90 days; $p<0.001$ for both). Post-index date direct allcause healthcare costs were higher for cases than for controls (median total cost: $\$ 18,428$ vs. $\$ 621$ for 30 days; $\$ 21,774$ vs. $\$ 3312$ for 90 days; $\$ 25,960$ vs. $\$ 8699$ for 6 months; $\$ 35,875$ vs. $\$ 21,619$ for 1 year; all $p<0.001)$.

Conclusions: Pneumonia as a complication of influenza increases risk of mortality and leads to greater healthcare resource use and direct medical costs among patients hospitalized with influenza. These effects are seen early during the index hospitalization and within the first 30 days after diagnosis, but their impact continues throughout a year of follow-up.

Keywords: Influenza; Pneumonia; Complication; Commercial; Retrospective; Health care resource use; Costs; Hospitalization 


\section{Key Summary Points}

Why carry out this study?

Influenza is an increasingly prevalent acute respiratory infection, and pneumonia is the most frequent serious complication.

Together, influenza and pneumonia are the eighth leading cause of death in the United States, but there is a lack of realworld studies focusing on the burden of pneumonia as a complication of influenza.

The purpose of this large retrospective observational study was to quantify the burden of pneumonia among hospitalized patients with influenza.

\section{What was learned from this study?}

Pneumonia as a complication of influenza increases risk of mortality and leads to greater healthcare resource use and direct medical costs among patients hospitalized with influenza.

These findings demonstrate the need for treatments that reduce influenza complications, especially in the 30 days after an influenza diagnosis.

\section{INTRODUCTION}

Influenza is an acute respiratory infection, having affected 9.2 million to 35.6 million people in the United States from the 2010-2011 to the 2015-2016 influenza season, resulting in increased morbidity and mortality [1]. Most of the resulting morbidity and mortality in influenza patients is due to complications disproportionately affecting vulnerable groups such as individuals with chronic disease (including chronic pulmonary disease), individuals aged $\geq 50$ years, pregnant women, children aged 6-59 months, individuals receiving

aspirin- or salicylate-containing medications, extremely obese individuals, and immunecompromised individuals [2, 3].

Pneumonia, either bacterial or viral, is the most frequent serious complication and usually occurs towards the resolution of influenza symptoms 4-14 days after diagnosis [4, 5]. Together, influenza and pneumonia are the eighth leading cause of death in the United States [6]. Respiratory-related deaths from the 2010-2011 to the 2013-2014 influenza season ranged from 12,000 to 56,000 . Pneumonia-associated and influenza deaths ranged from 4000-12,000 between the 2010-2011 and the 2015-2016 influenza season [1].

Complications from influenza resulting in hospitalization remain a significant health concern. During the 2017-2018 influenza season, the US Centers for Disease Control and Prevention (CDC) estimates there were $>45$ million cases of influenza resulting in $>21$ million influenza-associated medical visits, $>810,000$ influenza-related hospitalizations, and $>61,000$ deaths [7]. Along with the physical burden of influenza, there is also a significant economic burden. Previously, the projected annual cost of influenza-related healthcare has been estimated to range from $\$ 2.0-\$ 5.8$ billion (for two of the most common sub-types) to $\$ 10.4$ billion $[8,9]$.

Previous studies either examined influenzarelated or pneumonia-related healthcare resource utilization (HCRU). Many of the studies attempting to quantify the burden of influenza do not calculate the burden of influenzaassociated pneumonia [10-16]. At the same time, many pneumonia studies evaluate the burden of pneumonia (not including those evaluating viral or bacterial pneumonia) regardless of the etiology $[17,18]$. More specifically, there is a lack of real-world studies focusing on the burden of pneumonia as a complication of influenza-the most frequently reported complication of influenza. The purpose of this study was to quantify the burden of pneumonia among hospitalized patients with influenza. 


\section{METHODS}

\section{Data Source}

This real-world observational study was conducted using the Optum de-identified Clinformatics ${ }^{\circledR}$ Data Mart Database (2007-2020) ("Optum") from January 2014 through June 2019. The Optum database captures administrative claims for $>33$ million privately insured members with both medical and pharmacy benefits.

\section{Compliance with Ethics Guidelines}

Optum claims data files that were used for this study included medical claims, pharmacy claims, and laboratory results. All patient identifiers in the database have been fully encrypted, and the database is fully compliant with the Health Insurance Portability and Accountability Act (HIPAA) of 1996. As such, Institutional Review Board (IRB) approval to conduct this study was not required and considered exempt according to 45CFR46.101(b)(4): Existing Data $\&$ Specimens-No Identifiers.

\section{Patient Selection}

Patients with a medical claim for influenza diagnosis (International Classification of Disease, 9th Revision, Clinical Modification [ICD9-CM] codes 487, 488; ICD-10-CM codes J09, J10, J11) were identified between January 1, 2015 and June 30, 2018. The date of the first observed influenza diagnosis during this period was designated as the initial influenza diagnosis date. Patients were required to have $\geq 1$ allcause inpatient visit within 1 day of the initial influenza diagnosis date during the identification period. The first inpatient visit during this period was designated as the initial influenza hospitalization.

Patients were included in the case cohort if they had $\geq 1$ diagnosis for an influenza-related pneumonia (ICD-9-CM: 487.0, 488.01, 488.11, 488.81; ICD-10-CM: J09.X1, J10.0x, J11.0x) within the 30 days after the initial influenza diagnosis date during the identification period
(01JAN2015-30JUN2019). The first influenzarelated pneumonia diagnosis date was designated as the index date. Control patients were identified as patients with no evidence of influenza-related pneumonia in the 30 days following the initial influenza diagnosis. For each case patient, a control patient of identical age, sex, and region was selected during the same index study year. To reduce bias, distribution of time between initial influenza diagnoses and influenza-related pneumonia diagnoses for cases was calculated. The index date for controls was then randomly assigned based on the distribution of influenza-related pneumonia diagnoses to the index date for case patients.

Patients in both case and control cohorts were required to be $\geq 13$ years at the time of index, with continuous medical and pharmacy benefits for $\geq 12$ months prior to the index date (baseline period) and $\geq 12$ months after the index date (follow-up period). Patients who died during the follow-up period were also included in the study. To exclude patients with recurrent pneumonia leading up to the initial influenza diagnosis, patients were excluded if they had a diagnosis of pneumonia in the 30 days before the initial influenza diagnosis. Patients with recurrent pneumonia during the full baseline period were not excluded. (Fig. 1)

\section{Study Variables}

Baseline patient demographics including age, sex, US geographic region, health insurance plan type, and Charlson Comorbidity Index (CCI) score were evaluated $[19,20]$. Baseline comorbidities were assessed during the 12-month baseline period for all patients. Comorbidities of interest included ankylosing spondylitis, anxiety, asthma, cancer, chronic kidney disease, chronic liver disease, chronic obstructive pulmonary disease, congenital heart disease, congestive heart failure, coronary artery disease, Crohn's disease, cystic fibrosis, depression, diabetes, leukemia, high cholesterol, HIV, hypertension, lymphoma, multiple sclerosis, osteoarthritis, osteoporosis, plaque psoriasis, plasma cell neoplasms psoriatic arthritis, 


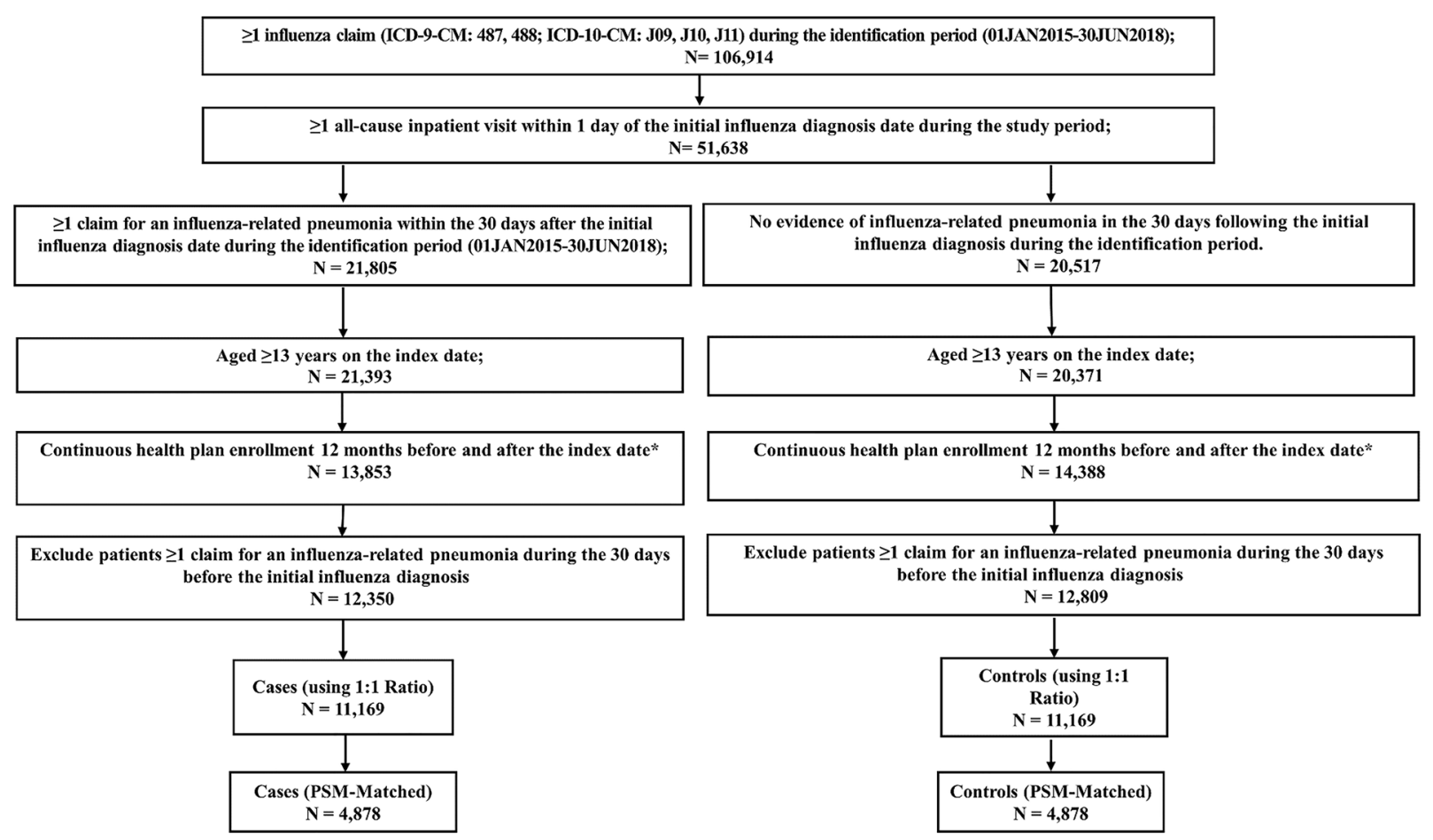

Fig. 1 Patient selection flow chart. ${ }^{*}$ Patients who died during the 12-month follow-up period were included in the analysis

rheumatoid arthritis, solid organ transplant, stem cell transplant, stroke, systemic lupus erythematosus, and ulcerative colitis. All comorbid conditions were identified using ICD9-CM or ICD-10-CM codes.

Characteristics of the initial influenza hospitalization were examined including the length of stay (LOS), intensive care unit (ICU) use, mechanical ventilation (MV) use, and initial influenza hospitalization healthcare costs. In addition, cumulative 30- and 90-day readmission as well as all-cause mortality during 30 days, 90 days, 6 months, and 12 months in the follow-up period were examined. All-cause HCRU and costs during the pre- and post-index period were assessed, including hospital LOS, office visits, pharmacy use, emergency department/room (ER), inpatient (across all hospitalization), and cumulative outpatient visits during 30 days, 90 days, 6 months, and 12 months in the follow-up. Costs were adjusted to 2019 US dollars using the medical care component of the Consumer Price Index.

\section{Statistical Methods}

All baseline and outcome variables were analyzed descriptively and compared between cases and controls. Propensity score matching (PSM) was performed to adjust for observed differences between the case and control cohorts. An unconditional logistic regression was fitted to determine the baseline characteristics associated with being in the case or control cohort. Using this model, a propensity score was developed for each patient which characterized the probability of being a member of the case cohort. Covariates in the propensity model included baseline demographic and clinical characteristics such as age, sex, US geographic region, health plan, payer type, pre-index CCI score, baseline all-cause HCRU, and individual comorbidities. Each patient in the case cohort was matched to a patient in the control cohort with the closest propensity score. Percentages and counts were provided for categorical variables. Means and standard deviations (SDs) were 
Table 1 Baseline demographics, clinical characteristics, and health care utilization and costs among hospitalized influenza patients with vs. without influenza-related pneumonia: before and after PSM

\begin{tabular}{|c|c|c|c|c|c|c|c|c|c|c|}
\hline & \multicolumn{5}{|c|}{ Before PSM } & \multicolumn{5}{|c|}{ After PSM } \\
\hline & \multicolumn{2}{|c|}{$\begin{array}{l}\text { Cases } \\
(N=11,169)\end{array}$} & \multicolumn{2}{|c|}{$\begin{array}{l}\text { Controls } \\
(N=11,169)\end{array}$} & \multirow[t]{2}{*}{$p$ value } & \multicolumn{2}{|c|}{$\begin{array}{l}\text { Cases } \\
(N=4878)\end{array}$} & \multicolumn{2}{|c|}{$\begin{array}{l}\text { Controls } \\
(N=4878)\end{array}$} & \multirow[t]{2}{*}{$p$ value } \\
\hline & $\bar{N}$ & $\%$ & $\bar{N}$ & $\%$ & & $\bar{N}$ & $\%$ & $N$ & $\%$ & \\
\hline \multicolumn{11}{|l|}{ Age on index date (years) } \\
\hline Mean $\pm S D$ & 76.4 & 11.1 & 76.4 & 11.1 & 0.858 & 78.4 & 9.0 & 78.4 & 8.9 & 0.841 \\
\hline Median & 78.0 & & 78.0 & & & 80.0 & & 80.0 & & \\
\hline \multicolumn{11}{|l|}{ Sex } \\
\hline Male & 4794 & $42.9 \%$ & 4794 & $42.9 \%$ & 1.000 & 2043 & $41.9 \%$ & 2043 & $41.9 \%$ & 1.000 \\
\hline Female & 6375 & $57.1 \%$ & 6375 & $57.1 \%$ & 1.000 & 2835 & $58.1 \%$ & 2835 & $58.1 \%$ & 1.000 \\
\hline \multicolumn{11}{|l|}{ Health plan type } \\
\hline Indemnity & 197 & $1.8 \%$ & 268 & $2.4 \%$ & 0.001 & 25 & $0.5 \%$ & 25 & $0.5 \%$ & 1.000 \\
\hline $\begin{array}{l}\text { Non-capitated point of } \\
\text { service }\end{array}$ & 753 & $6.7 \%$ & 781 & $7.0 \%$ & 0.459 & 186 & $3.8 \%$ & 186 & $3.8 \%$ & 1.000 \\
\hline $\begin{array}{l}\text { Health maintenance } \\
\text { organization }\end{array}$ & 3059 & $27.4 \%$ & 3053 & $27.3 \%$ & 0.928 & 1215 & $24.9 \%$ & 1215 & $24.9 \%$ & 1.000 \\
\hline $\begin{array}{l}\text { Preferred provider } \\
\text { organization }\end{array}$ & 828 & $7.4 \%$ & 886 & $7.9 \%$ & 0.145 & 204 & $4.2 \%$ & 204 & $4.2 \%$ & 1.000 \\
\hline $\begin{array}{l}\text { Exclusive provider } \\
\text { organization }\end{array}$ & 99 & $0.9 \%$ & 128 & $1.1 \%$ & 0.053 & 10 & $0.2 \%$ & 10 & $0.2 \%$ & 1.000 \\
\hline Others & 6233 & $55.8 \%$ & 6053 & $54.2 \%$ & 0.016 & 3238 & $66.4 \%$ & 3238 & $66.4 \%$ & 1.000 \\
\hline \multicolumn{11}{|l|}{ Payer Type } \\
\hline Medicare & 9948 & $89.1 \%$ & 9794 & $87.7 \%$ & 0.001 & 4639 & $95.1 \%$ & 4639 & $95.1 \%$ & 1.000 \\
\hline Commercial & 1221 & $10.9 \%$ & 1375 & $12.3 \%$ & 0.001 & 239 & $4.9 \%$ & 239 & $4.9 \%$ & 1.000 \\
\hline \multicolumn{11}{|l|}{ US geographic region } \\
\hline Northeast & 1693 & $15.2 \%$ & 1693 & $15.2 \%$ & 1.000 & 673 & $13.8 \%$ & 673 & $13.8 \%$ & 1.000 \\
\hline North Central & 3076 & $27.5 \%$ & 3066 & $27.5 \%$ & 0.881 & 1414 & $29.0 \%$ & 1414 & $29.0 \%$ & 1.000 \\
\hline South & 4164 & $37.3 \%$ & 4167 & $37.3 \%$ & 0.967 & 1851 & $37.9 \%$ & 1851 & $37.9 \%$ & 1.000 \\
\hline West & 2227 & $19.9 \%$ & 2232 & $20.0 \%$ & 0.933 & 940 & $19.3 \%$ & 940 & $19.3 \%$ & 1.000 \\
\hline Unknown & 9 & $0.1 \%$ & 11 & $0.1 \%$ & 0.655 & 0 & $0.0 \%$ & 0 & $0.0 \%$ & \\
\hline \multicolumn{11}{|c|}{ Charlson comorbidity index score } \\
\hline Mean \pm SD & 3.9 & 3.1 & 3.6 & 3.0 & $<0.001$ & 3.1 & 2.7 & 3.1 & 2.7 & 1.000 \\
\hline Median & 3.0 & & 3.0 & & & 2.0 & & 2.0 & & \\
\hline \multicolumn{11}{|l|}{ Baseline comorbidities } \\
\hline Anxiety & 2093 & $18.9 \%$ & 2085 & $18.9 \%$ & 0.929 & 807 & $16.5 \%$ & 828 & $17.0 \%$ & 0.569 \\
\hline Asthma & 1779 & $16.1 \%$ & 1727 & $15.6 \%$ & 0.361 & 632 & $13.0 \%$ & 675 & $13.8 \%$ & 0.201 \\
\hline
\end{tabular}


Table 1 continued

\begin{tabular}{|c|c|c|c|c|c|c|c|c|c|c|}
\hline & \multicolumn{5}{|c|}{ Before PSM } & \multicolumn{5}{|c|}{ After PSM } \\
\hline & \multicolumn{2}{|c|}{$\begin{array}{l}\text { Cases } \\
(N=11,169)\end{array}$} & \multicolumn{2}{|c|}{$\begin{array}{l}\text { Controls } \\
(N=11,169)\end{array}$} & \multirow[t]{2}{*}{$p$ value } & \multicolumn{2}{|c|}{$\begin{array}{l}\text { Cases } \\
(N=4878)\end{array}$} & \multicolumn{2}{|c|}{$\begin{array}{l}\text { Controls } \\
(N=4878)\end{array}$} & \multirow[t]{2}{*}{$p$ value } \\
\hline & $N$ & $\%$ & $N$ & $\%$ & & $N$ & $\%$ & $N$ & $\%$ & \\
\hline Cancer & 1907 & $17.2 \%$ & 1635 & $14.8 \%$ & $<.001$ & 585 & $12.0 \%$ & 578 & $11.8 \%$ & 0.827 \\
\hline $\begin{array}{l}\text { Chronic kidney disease } \\
\text { (CKD) }\end{array}$ & 3431 & $31.0 \%$ & 3268 & $29.6 \%$ & 0.020 & 1238 & $25.4 \%$ & 1294 & $26.5 \%$ & 0.196 \\
\hline $\begin{array}{l}\text { Chronic obstructive } \\
\text { pulmonary disease } \\
\text { (COPD) }\end{array}$ & 4595 & $41.5 \%$ & 4205 & $38.1 \%$ & $<0.001$ & 1796 & $36.8 \%$ & 1750 & $35.9 \%$ & 0.333 \\
\hline $\begin{array}{l}\text { Congestive heart failure } \\
(\mathrm{CHF})\end{array}$ & 3456 & $31.2 \%$ & 3169 & $28.7 \%$ & $<0.001$ & 1164 & $23.9 \%$ & 1164 & $23.9 \%$ & 1.000 \\
\hline $\begin{array}{l}\text { Coronary artery disease } \\
(\mathrm{CAD})\end{array}$ & 3712 & $33.6 \%$ & 3587 & $32.5 \%$ & 0.086 & 1491 & $30.6 \%$ & 1481 & $30.4 \%$ & 0.826 \\
\hline Depression & 2457 & $22.2 \%$ & 2369 & $21.4 \%$ & 0.168 & 955 & $19.6 \%$ & 901 & $18.5 \%$ & 0.164 \\
\hline Diabetes & 4130 & $37.3 \%$ & 4086 & $37.0 \%$ & 0.593 & 1634 & $33.5 \%$ & 1656 & $33.9 \%$ & 0.638 \\
\hline High cholesterol & 2322 & $21.0 \%$ & 2310 & $20.9 \%$ & 0.884 & 975 & $20.0 \%$ & 989 & $20.3 \%$ & 0.724 \\
\hline Hypertension & 9031 & $81.6 \%$ & 8987 & $81.3 \%$ & 0.582 & 3847 & $78.9 \%$ & 3892 & $79.8 \%$ & 0.261 \\
\hline Osteoarthritis & 3776 & $34.1 \%$ & 3761 & $34.0 \%$ & 0.888 & 1570 & $32.2 \%$ & 1571 & $32.2 \%$ & 0.983 \\
\hline Osteoporosis & 1445 & $13.1 \%$ & 1315 & $11.9 \%$ & 0.009 & 368 & $7.5 \%$ & 368 & $7.5 \%$ & 1.000 \\
\hline Stroke & 1093 & $9.9 \%$ & 1079 & $9.8 \%$ & 0.777 & 411 & $8.4 \%$ & 397 & $8.1 \%$ & 0.607 \\
\hline \multicolumn{11}{|c|}{ Baseline all-cause healthcare resource utilization } \\
\hline \multicolumn{11}{|l|}{ Hospitalizations } \\
\hline $\begin{array}{l}\text { Patients with } \geq 1 \\
\text { hospitalization }(n, \%)\end{array}$ & 4900 & $43.9 \%$ & 5731 & $51.3 \%$ & $<0.001$ & 1843 & $37.8 \%$ & 1843 & $37.8 \%$ & 1.000 \\
\hline $\begin{array}{l}\text { Hospitalizations per } \\
\text { patients (mean, SD) }\end{array}$ & 1.0 & 1.8 & 1.1 & 1.6 & 0.014 & 0.8 & 1.5 & 0.8 & 1.4 & 0.531 \\
\hline \multicolumn{11}{|l|}{ Outpatient Office visits } \\
\hline $\begin{array}{l}\text { Patients with } \geq 1 \\
\text { outpatient visit }(n, \%)\end{array}$ & 10,442 & $93.5 \%$ & 10,248 & $91.8 \%$ & $<0.001$ & 4,497 & $92.2 \%$ & 4,501 & $92.3 \%$ & 0.880 \\
\hline $\begin{array}{l}\text { Outpatient visits per } \\
\text { patients (mean, SD) }\end{array}$ & 10.2 & 8.6 & 9.6 & 8.1 & $<0.001$ & 8.9 & 7.6 & 8.8 & 7.3 & 0.431 \\
\hline \multicolumn{11}{|l|}{ Outpatient ER visits } \\
\hline $\begin{array}{l}\text { Patients with } \geq 1 \\
\text { outpatient ER visit }(n, \%)\end{array}$ & 6741 & $60.4 \%$ & 6577 & $58.9 \%$ & 0.025 & 2608 & $53.5 \%$ & 2608 & $53.5 \%$ & 1.000 \\
\hline $\begin{array}{l}\text { Outpatient ER visits per } \\
\text { patients (mean, SD) }\end{array}$ & 2.0 & 3.5 & 1.8 & 3.1 & $<0.001$ & 1.6 & 2.8 & 1.5 & 2.7 & 0.061 \\
\hline Pharmacy fills & & & & & & & & & & \\
\hline
\end{tabular}


Table 1 continued

\begin{tabular}{|c|c|c|c|c|c|c|c|c|c|c|}
\hline & \multicolumn{5}{|c|}{ Before PSM } & \multicolumn{5}{|c|}{ After PSM } \\
\hline & \multicolumn{2}{|c|}{$\begin{array}{l}\text { Cases } \\
(N=11,169)\end{array}$} & \multicolumn{2}{|c|}{$\begin{array}{l}\text { Controls } \\
(N=11,169)\end{array}$} & \multirow[t]{2}{*}{$p$ value } & \multicolumn{2}{|c|}{$\begin{array}{l}\text { Cases } \\
(N=4878)\end{array}$} & \multicolumn{2}{|c|}{$\begin{array}{l}\text { Controls } \\
(N=4878)\end{array}$} & \multirow[t]{2}{*}{$p$ value } \\
\hline & $N$ & $\%$ & $N$ & $\%$ & & $N$ & $\%$ & $N$ & $\%$ & \\
\hline $\begin{array}{l}\text { Patients with } \geq 1 \\
\text { pharmacy fills }(n, \%)\end{array}$ & 9541 & $85.4 \%$ & 9500 & $85.1 \%$ & 0.439 & 4032 & $82.7 \%$ & 4100 & $84.1 \%$ & 0.065 \\
\hline $\begin{array}{l}\text { Fills per patients (mean, } \\
\text { SD) }\end{array}$ & 27.1 & 24.5 & 25.6 & 23.1 & $<0.001$ & 23.9 & 22.5 & 23.4 & 21.3 & 0.242 \\
\hline \multicolumn{11}{|c|}{ Baseline all-cause healthcare costs } \\
\hline Inpatient costs & $\$ 18,079$ & $\$ 57,455$ & $\$ 17,115$ & $\$ 44,214$ & $<0.001$ & $\$ 13,379$ & $\$ 51,461$ & $\$ 12,196$ & $\$ 36,859$ & 0.192 \\
\hline Outpatient costs & $\$ 27,143$ & $\$ 75,008$ & $\$ 20,638$ & $\$ 52,934$ & $<0.001$ & $\$ 19,341$ & $\$ 56,112$ & $\$ 16,474$ & $\$ 44,947$ & 0.005 \\
\hline Pharmacy costs & $\$ 5,742$ & $\$ 15,995$ & $\$ 4931$ & $\$ 13,274$ & $<0.001$ & $\$ 4129$ & $\$ 11,133$ & $\$ 3859$ & $\$ 10,592$ & 0.220 \\
\hline Total costs & $\$ 50,964$ & $\$ 104,524$ & $\$ 42,684$ & $\$ 77,864$ & $<0.001$ & $\$ 36,848$ & $\$ 84,006$ & $\$ 32,529$ & $\$ 65,532$ & 0.005 \\
\hline \multicolumn{11}{|c|}{ Characteristics of index hospitalization } \\
\hline LOS (mean, SD) & 6.5 & 7.5 & 1.7 & 3.6 & $<0.001$ & 6.5 & 8.3 & 1.9 & 3.7 & $<0.001$ \\
\hline Index costs (mean, SD) & $\$ 18,300$ & $\$ 48,662$ & $\$ 3,933$ & $\$ 19,781$ & $<0.001$ & $\$ 18,221$ & $\$ 56,143$ & $\$ 4,478$ & $\$ 23,539$ & $<0.001$ \\
\hline ICU use $(n, \%)$ & 4476 & $40.1 \%$ & 1599 & $14.3 \%$ & $<0.001$ & 1875 & $38.4 \%$ & 771 & $15.8 \%$ & $<0.001$ \\
\hline MV use $(n, \%)$ & 2160 & $19.3 \%$ & 493 & $4.4 \%$ & $<0.001$ & 887 & $18.2 \%$ & 239 & $4.9 \%$ & $<0.001$ \\
\hline Invasive MV & 1362 & $12.2 \%$ & 249 & $2.2 \%$ & $<0.001$ & 556 & $11.4 \%$ & 111 & $2.3 \%$ & $<0.001$ \\
\hline Non-invasive MV & 798 & $7.1 \%$ & 244 & $2.2 \%$ & $<0.001$ & 331 & $6.8 \%$ & 128 & $2.6 \%$ & $<0.001$ \\
\hline
\end{tabular}

$L O S$ length of stay, $I C U$ intensive care unit, $M V$ mechanical ventilation, $E R$ emergency department/room, $S D$ standard deviation $p$ values were calculated using Student's $t$ tests and Pearson's Chi-squared tests for continuous and categorical variables, respectively

computed for continuous variables. Student's $t$ tests and Pearson's Chi-squared tests were used to test statistical significance for continuous and categorical variables, respectively, between the cohorts.

Statistical analyses were conducted using Statistical Analysis System (SAS) v.9.3. (Cary, NC, USA). The $p$ values level of significance was set at $\alpha$-level 0.05 .

\section{RESULTS}

\section{Baseline Characteristics}

There was a total of 106,914 patients aged $\geq 13$ years old with a diagnosis of influenza during the identification period
(01JAN2015-30JUN2018). A total of 51,638 (48\%) patients were hospitalized within 0-1 days of being diagnosed with influenza in the Optum database. After applying the inclusion and exclusion criteria, the final analytical sample was composed of a total of 25,159 patients including 12,350 influenza-related pneumonia cases $(49.1 \%)$ and 12,809 potential controls (50.9\%) without an influenza-related pneumonia diagnosis in the 30 days after the initial influenza diagnosis. After performing a 1:1 hard matching ratio based on age, gender, and region, there was a total of 11,169 patients with influenza-related pneumonia cases and 11,169 controls.

Pre-PSM patient characteristics and baseline HCRU and costs are summarized in Table 1. Prior to PSM, the average age among influenza- 
Table 2 PSM-adjusted mortality and hospital readmission during the follow-up among hospitalized influenza patients with vs. without influenza-related pneumonia

\begin{tabular}{|c|c|c|c|c|c|}
\hline & \multicolumn{2}{|c|}{$\begin{array}{l}\text { Cases } \\
N=\mathbf{4 8 7 8}\end{array}$} & \multicolumn{2}{|c|}{$\begin{array}{l}\text { Controls } \\
N=4878\end{array}$} & \multirow[t]{2}{*}{$P$ value } \\
\hline & $\bar{N}$ & $\%$ & $\bar{N}$ & $\%$ & \\
\hline \multicolumn{6}{|l|}{ Mortality } \\
\hline 30-day & 360 & $7.4 \%$ & 44 & $0.9 \%$ & $<.001$ \\
\hline 90-day & 699 & $14.3 \%$ & 143 & $2.9 \%$ & $<.001$ \\
\hline 6-month & 836 & $17.1 \%$ & 209 & $4.3 \%$ & $<.001$ \\
\hline 1 -year & 1,021 & $20.9 \%$ & 361 & $7.4 \%$ & $<.001$ \\
\hline \multicolumn{6}{|l|}{ Readmission } \\
\hline 30-day Readmission & 599 & $12.3 \%$ & 170 & $3.5 \%$ & $<.001$ \\
\hline 90-day Readmission Rate & 974 & $20.0 \%$ & 299 & $6.1 \%$ & $<.001$ \\
\hline
\end{tabular}

$p$ values were calculated using Student's $t$ tests and Pearson's Chi-squared tests for continuous and categorical variables, respectively

related pneumonia cases and controls was 76.4 years. Much of the total sample population were women residing in the south US region. Compared to controls, influenza-related pneumonia cases had higher mean CCI (3.9 vs. 3.6, $p<0.001)$. The most prevalent comorbidities for both cohorts included hypertension, chronic obstructive pulmonary disease, diabetes, osteoarthritis, coronary artery disease, and chronic kidney disease. Baseline all-cause HCRU were slightly higher among influenzarelated pneumonia cases than controls. On average, total baseline all-cause healthcare costs were higher among influenza-related pneumonia cases than controls $(\$ 50,964$ vs. $\$ 42,684$, $p<0.001)$. After 1:1 PSM, most baseline characteristics were well balanced between the study cohorts, with 4878 patients in each cohort (Table 1).

\section{Initial Influenza Hospitalization}

The characteristics of patients' initial influenza hospitalization are presented in Table 2. Compared to controls, influenza-related pneumonia cases had longer initial influenza hospitalization (6.5 days vs. 1.9, $p<0.001$ ), higher initial influenza hospitalization costs $(\$ 18,221$ vs.
$\$ 4478, p<0.001)$, higher proportion of ICU use (38.4 vs. $15.8 \%, p<0.001)$, higher MV use (18.2 vs. $4.9 \%, p<0.001)$, higher invasive MV (11.4 vs. $2.3 \%, p<0.001)$, and higher non-invasive MV use $(6.8$ vs. $2.6 \%, p<0.001)$ during the initial influenza hospitalization.

\section{Outcome Assessment}

Mortality and hospital readmission trends are presented in Table 3. Compared with controls, influenza-related pneumonia case patients also had higher mortality rates at 30 days ( 7.4 vs. $0.9 \%, p<0.001), 90$ days $(14.3$ vs. $2.9 \%$, $p<0.001), 6$ months (17.1 vs. $4.3 \%, p<0.001)$, and 1 year (20.9 vs. $7.4 \%, p<0.001)$ after the influenza-related pneumonia diagnosis date or randomly selected index date. Readmission rate was also higher in influenza-related pneumonia cases when compared to controls at 30 days (12.3 vs. $3.5 \%, p<0.001)$ and 90 days (20.0 vs. $6.1 \%, p<0.001)$ after the influenza-related pneumonia diagnosis date or randomly selected index date. 
Table 3 PSM adjusted follow-up all-cause healthcare resource utilization among hospitalized influenza patients with vs. without influenza-related pneumonia

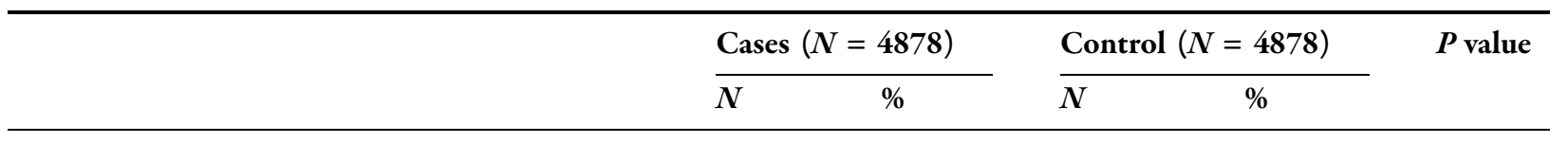

At 30 days

Hospitalizations

Patients with $\geq 1$ hospitalization $(n, \%)$

Hospitalizations per patients (mean, SD)

LOS, (mean, SD)

$\begin{array}{lllll}4578 & 93.8 \% & 784 & 16.1 \% & <0.001 \\ 1.2 & 0.7 & 0.2 & 0.5 & <0.001 \\ 12.3 & 18.0 & 1.8 & 8.6 & <0.001\end{array}$

Outpatient office visits

Patients with $\geq 1$ outpatient office visit $(n, \%)$

2721

$55.8 \%$

2363

$48.4 \%$

$<0.001$

Outpatient office visits per patients (mean, SD)

1.0

1.2

0.8

1.1

$<0.001$

Outpatient ER visits

Patients with $\geq 1$ outpatient ER visit $(n, \%)$

3716

$$
76.2 \%
$$

864

$17.7 \%$

$<0.001$

Outpatient ER visits per patients (mean, SD)

1.0

0.8

0.3

0.7

$<0.001$

Pharmacy fills

Patients with $\geq 1$ pharmacy fills $(n, \%)$

3289

$67.4 \%$

3376

$69.2 \%$

0.058

Fills per patients (mean, SD)

$$
2.2
$$

2.1

2.1

0.198

At 90 days

Hospitalizations

Patients with $\geq 1$ hospitalization $(n, \%)$

4617

$94.6 \%$

1440

$29.5 \%$

$<0.001$

Hospitalizations per patients (mean, SD)

1.4

1.0

0.4

0.8

$<.001$

LOS, (mean, SD)

14.7

22.4

4.0

12.9

$<0.001$

Outpatient office visits

Patients with $\geq 1$ outpatient office visit $(n, \%)$

3636

$74.5 \%$

3710

$76.1 \%$

0.082

Outpatient office visits per patients (mean, SD)

Outpatient ER visits

Patients with $\geq 1$ outpatient ER visit $(n, \%)$

3863

$79.2 \%$

1646

$33.7 \%$

$<0.001$

Outpatient ER visits per patients (mean, SD)

1.3

0.6

1.2

$<0.001$

Pharmacy fills

Patients with $\geq 1$ pharmacy fills $(n, \%)$

3660

$75.0 \%$

3866

$79.3 \%$

$<0.001$

Fills per patients (mean, SD)

5.8

5.7

6.0

5.6

0.049

At 6 months

Hospitalizations

Patients with $\geq 1$ hospitalization $(n, \%)$

4642

$95.2 \%$

1807

$37.0 \%$

$<0.001$ 
Table 3 continued

\begin{tabular}{|c|c|c|c|c|c|}
\hline & \multicolumn{2}{|c|}{ Cases $(N=4878)$} & \multicolumn{2}{|c|}{ Control $(N=4878)$} & \multirow[t]{2}{*}{$P$ value } \\
\hline & $\bar{N}$ & $\%$ & $\bar{N}$ & $\%$ & \\
\hline Hospitalizations per patients (mean, SD) & 1.6 & 1.3 & 0.6 & 1.1 & $<0.001$ \\
\hline LOS, (mean, SD) & 16.7 & 26.0 & 6.1 & 16.9 & $<.001$ \\
\hline
\end{tabular}

Outpatient office visits

Patients with $\geq 1$ outpatient office visit $(n, \%)$

$\begin{array}{lllll}3857 & 79.1 \% & 4170 & 85.5 \% & <0.001 \\ 4.7 & 4.5 & 4.6 & 4.3 & 0.446 \\ & & & & \\ 4011 & 82.2 \% & 2160 & 44.3 \% & <0.001 \\ 1.6 & 1.8 & 1.0 & 1.8 & <0.001 \\ & & & & <0.001 \\ 3733 & 76.5 \% & 3,974 & 81.5 \% & 0.003\end{array}$

Fills per patients (mean, SD)

At 12 months

\section{Hospitalizations}

Patients with $\geq 1$ hospitalization $(n, \%)$

Hospitalizations per patients (mean, SD)

LOS, (mean, SD)

$\begin{array}{lllll}4679 & 95.9 \% & 2762 & 56.6 \% & <0.001 \\ 2.0 & 1.8 & 1.2 & 1.6 & <0.001 \\ 20.4 & 31.0 & 11.6 & 26.9 & <0.001\end{array}$

Outpatient office visits

Patients with $\geq 1$ outpatient office visit $(n, \%)$

$\begin{array}{lllll}3961 & 81.2 \% & 4373 & 89.6 \% & <0.001\end{array}$

Outpatient office visits per patients (mean, SD)

$8.6 \quad 8$

Outpatient ER visits

Patients with $\geq 1$ outpatient ER visit $(n, \%)$

$\begin{array}{lllll}4183 & 85.8 \% & 3091 & 63.4 \% & <0.001 \\ 2.4 & 3.1 & 2.0 & 3.0 & <0.001\end{array}$

Outpatient ER visits per patients (mean, SD)

2.4

8.3

9.0

7.8

0.007

Pharmacy fills

Patients with $\geq 1$ pharmacy fills $(n, \%)$

Fills per patients (mean, SD)

\begin{tabular}{lllll}
3814 & $78.2 \%$ & 4116 & $84.4 \%$ & $<0.001$ \\
19.8 & 20.6 & 21.7 & 20.3 & $<0.001$ \\
\hline
\end{tabular}

$E R$ emergency department/room, $L O S$ length of stay, $S D$ standard deviation

$p$ values were calculated using Student's $t$ tests and Pearson's Chi-squared tests for continuous and categorical variables, respectively 


\section{All-cause Healthcare Utilization and Costs}

Healthcare resource utilization is summarized in Table 3. Compared to controls, influenza-related pneumonia cases had higher all-cause inpatient admissions (93.8 vs. $16.1 \%$ within 30 days; 94.6 vs. $29.5 \%$ within 90 days; 95.2 vs. $37.0 \%$ within 6 months; 95.9 vs. $56.6 \%$ within 1 year of the influenza-related pneumonia diagnosis date or the randomly selected index date; $p<0.001$ for all) and ER visits (76.2 vs. $17.7 \%$ within 30 days; 79.2 vs. $33.7 \%$ within 90 days; 82.2 vs. $44.3 \%$ within 6 months; 85.8 vs. $63.4 \%$ within 1 year of the influenza-related pneumonia diagnosis date or the randomly selected index date; $p<0.001$ for all). On average, cases had more inpatient admissions (1.2 vs. 0.2 within 30 days; 1.4 vs. 0.4 within 90 days; 1.6 vs. 0.6 within 6 months; 2.0 vs. 1.2 within 1 year post-index date; $p<0.001$ for all), more ER visits admissions (1.0 vs. 0.3 within 30 days; 1.3 vs. 0.6 within 90 days; 1.6 vs. 1.0 within 6 months; 2.4 vs. 2.0 within 1 year of the influenza-related pneumonia diagnosis date or the randomly selected index date; $p<0.001$ for all), and longer LOS (12.3 vs. 1.8 within 30 days; 14.7 vs. 4.0 within 90 days; 16.7 vs. 6.1 within 6 months; 20.4 vs. 11.6 within 1 year of the influenza-related pneumonia diagnosis date or the randomly selected index date; $p<0.001$ for all) per patient than controls. All healthcare utilization results were cumulative.

Figure 2 illustrates the differences in allcause healthcare costs during the follow-up. Average total all-cause healthcare costs post influenza-related pneumonia diagnosis date or the randomly selected index date among cases were higher when compared to controls ( $\$ 28,051$ vs. $\$ 6037$ for 30 days; $\$ 37,082$ vs. $\$ 13,309$ for 90 days; $\$ 46,835$ vs. $\$ 23,399$ for 6 months; $\$ 64,052$ vs. $\$ 43,976$ for 1 year; $p<0.001$ for all). The major costs driver was predominantly in the inpatient setting where influenza-related pneumonia cases incurred $\$ 19,740$ more than controls within 30 days, $\$ 20,896$ within 90 days, $\$ 20,319$ within 6 months and $\$ 18,401$ within 1 year of followup (all $p<0.001$ ). All healthcare cost results were cumulative.

\section{DISCUSSION}

This real-world retrospective data study quantified the burden of pneumonia among hospitalized patients with influenza using a large US managed care population. Overall, $48 \%$ of influenza patients were initially hospitalized within 0-1 day of diagnosis. After controlling for baseline differences using PSM, the baseline characteristics were well balanced between hospitalized influenza patients with influenzarelated pneumonia (cases) versus influenza patients without (controls).

The findings of this study suggest that pneumonia as a complication of influenza increases the risk of mortality and hospital readmission and also leads to greater HCRU and direct medical costs among patients hospitalized with influenza. The impact of pneumonia on these outcomes was evident particularly during the index hospitalization and within the first 30 days after diagnosis; however, their effects continued during the course of the first year of follow-up.

In most influenza cases, the infection is a self-limiting disease that resolves on its own without the risk of developing serious complications [21-23]. However, most of the resulting morbidity and mortality in influenza patients is due to complications, with pneumonia being the most prevalent complication of influenza $[5,24]$. The average age of this study population was 76 years, which is consistent with previous findings that adults aged $\geq 65$ years are at high risk of developing pneumonia and being hospitalized $[5,10,25]$. This risk, as well as the risk for subsequent reinfection and slow recovery, may remain elevated for several months. The current guidelines recommend both influenza and pneumococcal vaccines in patients aged $>65$ years [26]. However, the uptake for both vaccinations is low in this high-risk population [26]. The results of this study underscore the need to prevent and reduce the risk of pneumonia in the elderly population alongside the associated burden in mortality and morbidity.

During the index hospitalization, case patients with influenza-related pneumonia 


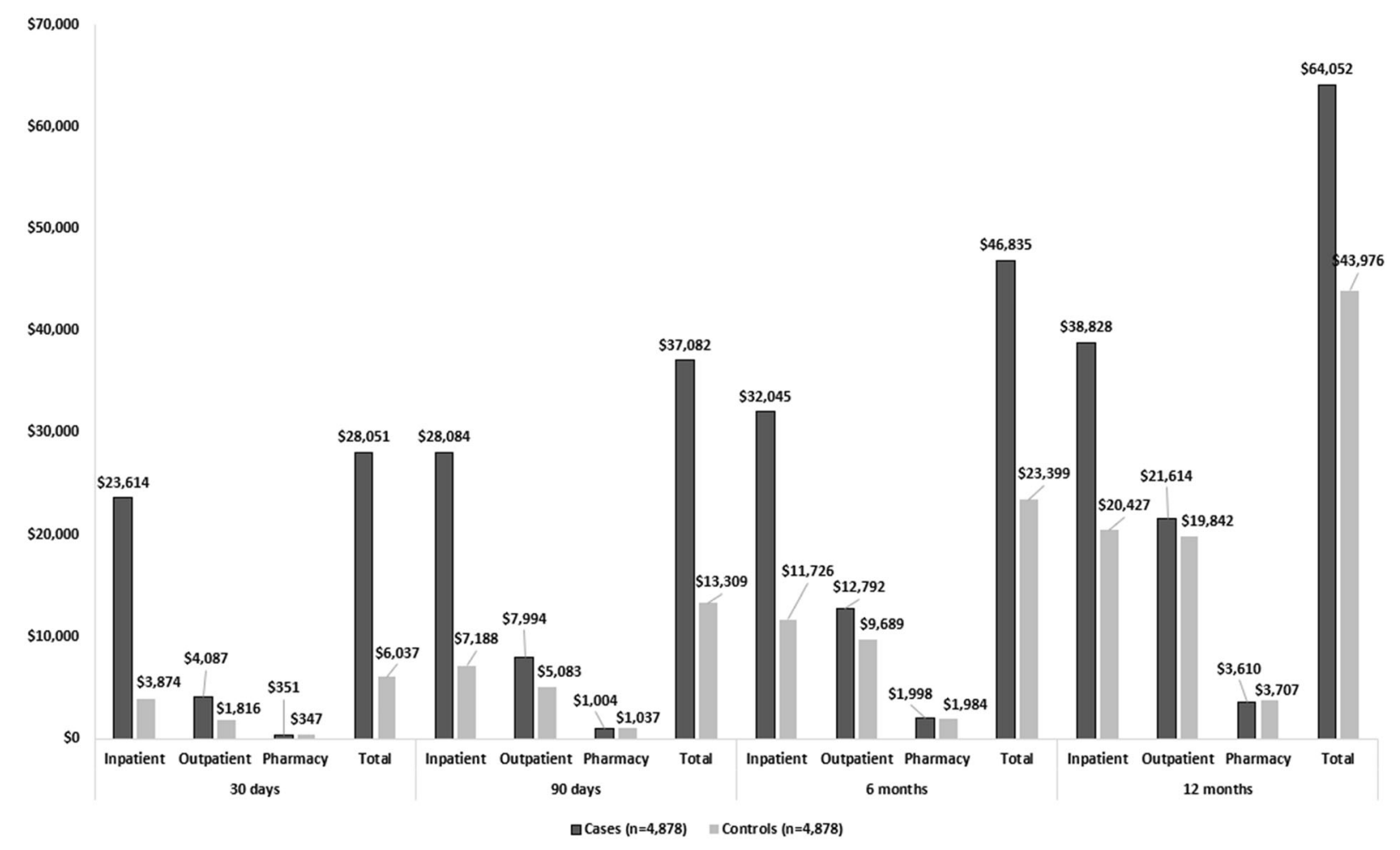

Fig. 2 PSM adjusted all-cause healthcare costs among hospitalized influenza patients with vs. without influenza-related pneumonia

remained in the hospital 5 days longer than controls; they were also more likely to be in the ICU and require MV and incurred more than $\$ 13,743$ compared to controls. These findings are consistent with studies that have demonstrated that the duration of influenza associated hospitalization averages 4-8 days: this is dependent of several factors including patient's age, the nature of complications and comorbidities, and whether patients require the use of MV, which may impact hospital LOS [27-30]. These factors potentially lead to increased economic burden.

In our study, we found significant 30-day readmission percentage differences between our cases and controls. Similar work calculating 30-day readmission among Canadian patients hospitalized with acute respiratory distress with laboratory-confirmed influenza calculated their 30 -day readmission to be $5.7 \%$ [31]. While our results seem different, the 30-day readmission of our pooled population is $7.9 \%$. The difference we are observing in our cohorts may be representative of the spectrum of influenza patients hospitalized and then readmitted.

It is known that influenza-related pneumonia has long been associated with increased morbidity and mortality $[25,29,32]$. In addition, deaths caused by influenza are usually due to complications including secondary pneumonia or worsening of an underlying condition. Previous studies have reported varying mortality rates from 11.78 per 100,000 personyears in the general population to 100 per 100,000 person-years in the Veterans Affairs population $[15,25]$. Mortality rates as high as 141.15 per 100,000 person-years have been noted for older adults aged $\geq 75$ compared to adults aged $65-74[10,25]$. Between October 2017 and January 2018, the weekly percentage of deaths attributed to pneumonia and influenza has ranged from 5.8 to $10.1 \%$ [33]. Both cases and controls cohorts in this study were elderly hospitalized influenza patients with a 
close comorbidity profile during the baseline period. However, having influenza-related pneumonia increased the risk of mortality by $\sim 7 \%$ during the first 30 days post influenzarelated pneumonia diagnosis. This risk remained elevated by the end of the first year of follow-up.

Previous studies have also focused exclusively on patients with influenza-related complications $[4,9,34]$. Our results were consistent with those of a previous study in which administrative claims were also used to analyze healthcare costs and HCRU between influenza patients with and without influenza-related complications [4]. Both healthcare costs and HCRU were found to be higher among influenza patients with complications and critical illnesses necessitating hospitalization [4]. Among influenza patients in large commercial claims database, Karve et al. estimated mean predicted total healthcare costs to be twice as high for influenza patients with influenza-related complications versus those without (\$3422 vs. $\$ 1510$ ) at the end of the 1-year follow-up period [4]. Our study found differences in costs between cases versus controls; however, our estimated costs were significantly higher for both cohorts ( $\$ 64,052$ vs. $\$ 43,976)$. It should be noted that Karve et al. did not require influenza patients to have an inpatient visit and did not restrict the timeframe for the outcome of the index influenza-related complication [4]. Additionally, their list of influenza-related complications was much more inclusive than ours and included various other conditions including acute otitis media, cardiovascular disease, and respiratory disorders [4].

Putri et al. focused on estimating the economic burden of influenza regardless of the incidence of complications [34]. The authors used previously published health outcome rates and age-specific symptomatic attack rates for seasonal influenza to estimate the direct and indirect costs of influenza, including the costs of hospitalizations among patients with specified influenza ICD codes [34]. Their mean estimated costs for hospitalized patients aged 65-84 (mean: \$8330) falls in between the mean costs of both cohorts' index hospitalization cost (mean: \$18,221 vs. \$4478) [34]. Subsequent costs for our case patients are higher, as these are the patients that developed pneumoniarequiring further HCRU.

To our knowledge, this is the largest retrospective observational study to compare the risk of ICU admission, mechanical ventilation use, hospital re-admission, mortality and economic burden in patients with a pneumonia diagnosis within 30 days of a hospitalization for influenza compared to patients who did not have a diagnosis of pneumonia in the 30 days after influenza hospitalization. There is a need for early and appropriate management of hospitalized influenza patients with influenza-related pneumonia. The findings from this study have demonstrated that complications resulting from influenza infection-particularly pneumoniacan lead to increased morbidity including longer hospital stay, ICU and MV utilization, risk of mortality, and increased HCRU and costs, especially in the elderly population. The need for early and appropriate management to avoid influenza-related complications has become even more emergent during the COVID-19 pandemic, which may place influenza patients in competition with severe acute respiratory syndrome coronavirus 2 (SARS-CoV2) patients for already scarce hospital resources.

\section{Limitations}

Our study had multiple limitations that need to be taken into consideration. While claims data are extremely valuable for the efficient and effective examination of healthcare outcomes, resource use, and costs, claims data are collected for the purpose of payment and not research. Therefore, certain limitations are associated with claims data use. Given the observational nature of the study, only associations without causal linkage can be inferred. Pneumonia diagnoses were captured based on ICD-9/ICD10 codes and not based on clinical parameters. The presence of an ICD-9/ICD-10 diagnosis code for pneumonia on a medical claim does not necessarily indicate the presence of the condition which may have resulted in patient misclassification. Influenza diagnosis was also identified using ICD-9/ICD-10 codes as opposed 
to laboratory data which could have led to the inclusion of influenza-like illness. Retrospective claims analyses are subject to coding errors or incorrectly entered diagnoses that were primarily coded for reimbursement purposes rather than clinical accuracy, therefore, some medical information may be unavailable or inaccurate.

\section{CONCLUSIONS}

Influenza-related pneumonia among those hospitalized with influenza led to an increased risk of mortality and greater HCRU and direct medical costs. These effects were seen early during the index hospitalization in the form of longer LOS and greater MV and ICU use and continued within the first 30 days after influenza diagnosis, but their impact remained throughout a year of follow-up. These findings demonstrate the need for treatments that reduce influenza complications-especially during the index hospitalization and in the 30 days after an influenza diagnosis.

\section{ACKNOWLEDGEMENTS}

Funding. This study was funded by Janssen Global Services, LLC. Rapid Service Fees were funded by Janssen Global Services, LLC.

Authorship. All authors had full access to all of the data in this study and take complete responsibility for the integrity of the data and accuracy of the data analysis. All named authors meet the International Committee of Medical Journal Editors (ICMJE) criteria for authorship for this article, take responsibility for the integrity of the work as a whole, and have given their approval for this version to be published.

Author Contributions. All authors contributed to the concept and design, statistical analysis, and drafting the manuscript.

Medical Writing, Editorial, and Other Assistance. Editorial assistance was provided by Chris Haddlesey and Michael Moriarty who are paid employees of SIMR, LLC, which is a paid consultant to Janssen Global Services, LLC.

Disclosures. Susan C. Bolge is an employee of Janssen Global Services, LLC, the study sponsor. Cynthia Gutierrez is an employee of SIMR, LLC, a paid consultant to the study sponsor. Furaha Kariburyo and Ding He were paid employees of SIMR, LLC at the time of study.

Compliance with Ethics Guidelines. Optum claims data files that were used for this study included medical claims, pharmacy claims, and laboratory results. All patient identifiers in the database have been fully encrypted, and the database is fully compliant with the Health Insurance Portability and Accountability Act (HIPAA) of 1996. As such, IRB approval to conduct this study was not required and considered exempt according to 45CFR46.101(b)(4): Existing Data \& Specimens-No Identifiers.

Data Availability. The raw data on which this analysis was based are not publicly available due to a data licensing agreement.

Open Access. This article is licensed under a Creative Commons Attribution-NonCommercial 4.0 International License, which permits any non-commercial use, sharing, adaptation, distribution and reproduction in any medium or format, as long as you give appropriate credit to the original author(s) and the source, provide a link to the Creative Commons licence, and indicate if changes were made. The images or other third party material in this article are included in the article's Creative Commons licence, unless indicated otherwise in a credit line to the material. If material is not included in the article's Creative Commons licence and your intended use is not permitted by statutory regulation or exceeds the permitted use, you will need to obtain permission directly from the copyright holder. To view a copy of this licence, visit http://creativecommons.org/licenses/by$\mathrm{nc} / 4.0 /$. 


\section{REFERENCES}

1. Rolfes MA, Foppa IM, Garg S, et al. Annual estimates of the burden of seasonal influenza in the United States: a tool for strengthening influenza surveillance and preparedness. Influenza Other Respir Viruses. 2018;12(1):132-7.

2. Gordon A, Reingold A. The burden of influenza: a complex problem. Curr Epidemiol Rep. 2018;5(1): $1-9$.

3. Grohskopf LA, Alyanak E, Broder KR, Walter EB, Fry AM, Jernigan DB. Prevention and control of seasonal influenza with vaccines: recommendations of the advisory committee on immunization practices-United States, 2019-20 influenza season. MMWR Recomm Rep. 2019;68(3):1-21.

4. Karve S, Misurski D, Herrera-Taracena G, Davis KL. Annual all-cause healthcare costs among influenza patients with and without influenza-related complications: analysis of a United States managed care database. Appl Health Econ Health Policy. 2013;11(2):119-28.

5. Rothberg MB, Haessler SD. Complications of seasonal and pandemic influenza. Crit Care Med. 2010;38(4 Suppl):e91-7.

6. Heron M. Deaths: leading causes for 2017. Natl Vital Stat Rep. 2019;68(6):1-77.

7. Garten R, Blanton L, Elal AI, et al. Update: influenza activity in the United States during the 2017-18 season and composition of the 2018-19 influenza vaccine. MMWR Morb Mortal Wkly Rep. 2018;67: 634-42.

8. Molinari NA, Ortega-Sanchez IR, Messonnier ML, et al. The annual impact of seasonal influenza in the US: measuring disease burden and costs. Vaccine. 2007;25(27):5086-96.

9. Yan S, Weycker D, Sokolowski S. US healthcare costs attributable to type A and type B influenza. Hum Vaccin Immunother. 2017;13(9):2041-7.

10. Czaja CA, Miller L, Alden N, et al. Age-related differences in hospitalization rates, clinical presentation, and outcomes among older adults hospitalized with influenza-U.S. Influenza Hospitalization Surveillance Network (FluSurv-NET). Open Forum Infect Dis. 2019;6(7):ofz225.

11. Klepser DG, Corn CE, Schmidt M, Dering-Anderson AM, Klepser ME. Health care resource utilization and costs for influenza-like illness among midwestern health plan members. J Manag Care Spec Pharm. 2015;21(7):568-73.
12. Lucero-Obusan C, Schirmer PL, Wendelboe A, et al. Epidemiology and burden of influenza in the U.S. Department of Veterans Affairs. Influenza Other Respir Viruses. 2018;12(2):293-8.

13. Luckhaupt SE, Sweeney $\mathrm{MH}$, Funk R, et al. Influenza-associated hospitalizations by industry, 2009-10 influenza season. United States Emerg Infect Dis. 2012;18(4):556-62.

14. Reed C, Chaves SS, Daily Kirley P, et al. Estimating influenza disease burden from population-based surveillance data in the United States. PLoS One. 2015;10(3):e0118369

15. Young-Xu Y, van Aalst R, Russo E, et al. The annual burden of seasonal influenza in the US Veterans Affairs population. PLoS One. 2017;12(1):e0169344.

16. Zhou H, Thompson WW, Belongia EA, et al. Estimated rates of influenza-associated outpatient visits during 2001-2010 in 6 US integrated healthcare delivery organizations. Influenza Other Respir Viruses. 2018;12(1):122-31.

17. Gu CH, Lucero DE, Huang CC, Daskalakis D, Varma JK, Vora NM. Pneumonia-associated hospitalizations, New York City, 2001-2014. Public Health Rep. 2018;133(5):584-92.

18. Hayes BH, Haberling DL, Kennedy JL, Varma JK, Fry AM, Vora NM. Burden of pneumonia-associated hospitalizations: United States, 2001-2014. Chest. 2018;153(2):427-37.

19. Quan H, Sundararajan V, Halfon P, et al. Coding algorithms for defining comorbidities in ICD-9-CM and ICD-10 administrative data. Med Care. 2005;43(11):1130-9.

20. Charlson ME, Charlson RE, Peterson JC, Marinopoulos SS, Briggs WM, Hollenberg JP. The Charlson comorbidity index is adapted to predict costs of chronic disease in primary care patients. J Clin Epidemiol. 2008;61(12):1234-40.

21. Mauskopf J, Klesse M, Lee S, Herrera-Taracena G. The burden of influenza complications in different high-risk groups: a targeted literature review. J Med Econ. 2013;16(2):264-77.

22. Ghebrehewet S, MacPherson P, Ho A. Influenza. BMJ. 2016;355:i6258.

23. Centers for Disease Control and Prevention. People at high risk for flu complications. Centers for Disease Control and Prevention website. Accessed 29 July 2020 https://www.cdc.gov/flu/about/disease/ high_risk.htm 
24. Rothberg MB, Haessler SD, Brown RB. Complications of viral influenza. Am J Med. 2008;121(4): 258-64.

25. Quandelacy TM, Viboud C, Charu V, Lipsitch M, Goldstein E. Age- and sex-related risk factors for influenza-associated mortality in the United States between 1997-2007. Am J Epidemiol. 2014;179(2): 156-67.

26. Simonetti AF, Viasus D, Garcia-Vidal C, Carratalà J. Management of community-acquired pneumonia in older adults. Ther Adv Infect Dis. 2014;2(1):3-16.

27. Thompson WW, Shay DK, Weintraub E, et al. Influenza-associated hospitalizations in the United States. JAMA. 2004;292(11):1333-40.

28. Lee N, Chan PK, Choi KW, et al. Factors associated with early hospital discharge of adult influenza patients. Antivir Ther. 2007;12(4):501-8.

29. Lee N, Choi KW, Chan PK, et al. Outcomes of adults hospitalised with severe influenza. Thorax. 2010;65(6):510-5.
30. Lee N, Ison MG. Diagnosis, management and outcomes of adults hospitalized with influenza. Antivir Ther. 2012;17(1 Pt B):143-57.

31. Ng C, Ye L, Noorduyn SG, et al. Resource utilization and cost of influenza requiring hospitalization in Canadian adults: a study from the serious outcomes surveillance network of the Canadian immunization research network. Influenza Other Respir Viruses. 2018;12(2):232-40.

32. Oliva J, Delgado-Sanz C, Larrauri A, et al. Estimating the burden of seasonal influenza in Spain from surveillance of mild and severe influenza disease, 2010-2016. Influenza Other Respir Viruses. 2018;12(1):161-70.

33. Budd AP, Wentworth DE, Blanton L, et al. Update: influenza activity-United States, October 1, 2017-February 3, 2018. MMWR Morb Mortal Wkly Rep. 2018;67:169-79.

34. Putri WCWS, Muscatello DJ, Stockwell MS, Newall AT. Economic burden of seasonal influenza in the United States. Vaccine. 2018;36(27):3960-6. 